\title{
Tumour necrosis factor receptor-75 and risk of COPD exacerbation in the azithromycin trial
}

\section{To the Editor:}

We recently found that azithromycin taken daily for 1 year reduced the frequency of acute exacerbations of chronic obstructive pulmonary disease (AECOPDs) in subjects who were selected as having an increased risk of exacerbations [1]. Since azithromycin has potentially adverse effects, identifying patients most likely to benefit might be useful for targeting therapy. Accordingly, we included a substudy in our trial that was prospectively designed to determine whether plasma levels at entry, or a change in levels at 3 months of any of four blood biomarkers, were associated with improved clinical response to azithromycin as compared to placebo. We chose to study biomarkers of inflammatory pathways likely to be relevant to chronic obstructive pulmonary disease (COPD) including C-reactive protein (CRP), interleukin (IL)-6, IL-8 and soluble tumour necrosis factor receptor 75 (sTNFR75).

The parent trial comprised 1142 subjects with COPD randomised to receive azithromycin (250 mg orally each day) or placebo (1:1) for 12 months (ClinicalTrials.gov NCT00325897) [1]. To enrich our population for subjects more likely to have an AECOPDs, we required that subjects used either systemic corticosteroids, visited an emergency room or were hospitalised for AECOPD in the preceding 12 months or were using continuous supplemental oxygen [2]. We required subjects to be free of AECOPDs or other acute illness for $\geqslant 4$ weeks prior to enrolment and excluded subjects with known congestive heart failure (CHF) and those with clinically defined bronchiectasis. The primary outcome was time to first AECOPD defined as "a complex of respiratory symptoms (increased or new onset) of more than one of the following: cough, sputum, wheezing, dyspnoea, or chest tightness with a duration of at least 3 days requiring treatment with antibiotics or systemic steroids" [2]. We monitored participants for AECOPDs at follow-up clinic visits at months 1, 3, 6, 9 and 12 and by monthly phone contacts.

We obtained plasma at enrolment and after 3 months of treatment, and performed ELISA for CRP, IL-6, IL-8 and sTNFR75. We used Cox proportional hazards models (stratified by clinic as per the parent study) to relate biomarker levels to time to first AECOPD and paired and unpaired t-tests on an intention-to-treat basis to determine whether azithromycin affected biomarker levels (natural-log transformed) as compared to placebo. We then controlled for the following potential confounders: age, sex, smoking status, symptoms of chronic bronchitis, use of oxygen, inhaled corticosteroids, baseline forced expiratory volume in $1 \mathrm{~s}$ (FEV1) \% predicted and compliance (by pill count). We used interaction terms (biomarker $\times$ treatment assignment) to identify biomarkers that were associated with a modification in risk for AECOPD by treatment.

We obtained blood samples at enrolment in 1037 (91\%) of the 1142 subjects in the parent trial [1] (41\% Global Initiative for Chronic Obstructive Lung Disease class III, 21\% current smokers and 47\% with chronic bronchitis defined by responses to the St George's Respiratory Questionnaire). Blood samples were also available in $890(78 \%)$ subjects at 3 months.

In the subjects who had biomarker analyses, taking azithromycin was associated with a longer time to first exacerbation than placebo (HR 0.71, p $<0.001$ ), which was similar to that found in the parent trial. Higher plasma sTNFR75 levels at enrolment were associated with decreased time to first exacerbation (HR 1.21 for each two-fold elevation in sTNFR75, $\mathrm{p}=0.020$ ) when stratifying by clinic. This finding persisted when controlling for age, sex, current smoking, use of oxygen or inhaled corticosteroids, initial FEV1 \% pred, presence of chronic bronchitis and compliance (HR 1.12 for each two-fold elevation in sTNFR75, p $=0.017$ ). No significant associations were observed with respect to CRP, IL-6 or IL-8 measured at enrolment and time to first exacerbation in either univariate or multivariate analyses (all $\mathrm{p}>0.05$ ).

sTNFR75 concentrations at enrolment did not predict response to azithromycin $(p=0.89$ for the interaction term on Cox-proportional hazard modelling while controlling for clinic, $\mathrm{p}=0.74$ while controlling for clinic and other potential confounders). However, in pre-specified analyses of change in biomarker levels over time, a decline in sTNFR75 concentrations at 3 months identified subjects who benefited from azithromycin after 3 months in that their time to first exacerbation was longer than that for 

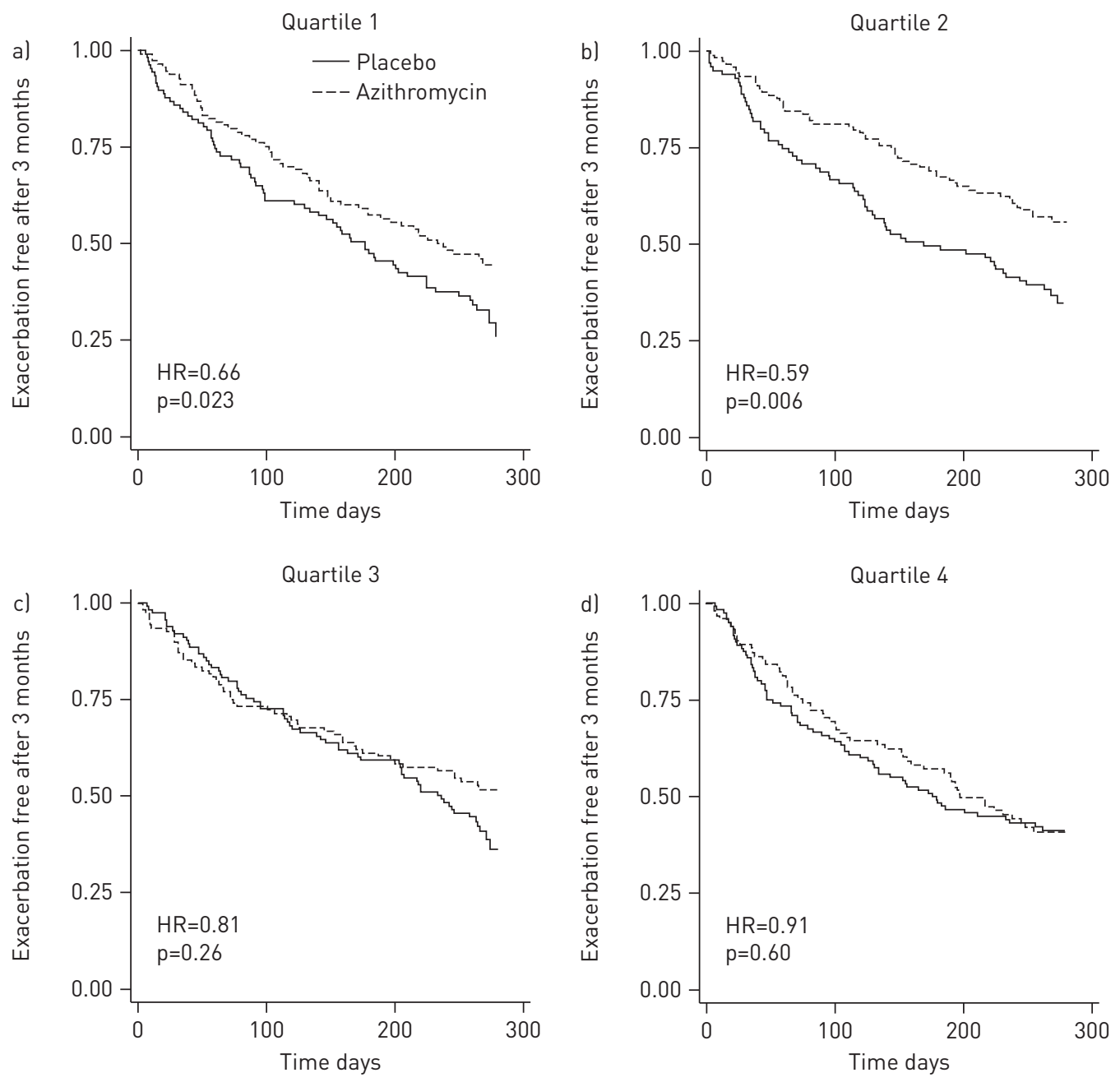

FIGURE 1 Effect of azithromycin on time to first exacerbation by change in soluble tumour necrosis factor-75 (sTNFR75) level at 3 months. 890 subjects had biomarker data at enrolment and at 3 months. a, b) Any decline in sTNFR75 levels between 0 and 3 months identified subjects in whom azithromycin was superior to placebo after 3 months. c, d) In subjects who did not manifest a decline in sTNFR75 levels, azithromycin was no better than placebo. Data are adjusted for centre. Findings persist while adjusting for centre and other potential confounders, e.g. age, sex, current smoking, oxygen use, inhaled corticosteroid use, forced expiratory volume in il s, bronchitis and compliance. Quartile 1: large decline in sTNFR75 at 3 months; quartile 2: moderate decline in sTNFR75 at 3 months; quartile 3: moderate increase in sTNFR75 at 3 months; quartile 4: large increase in sTNFR75 at 3 months. HR: hazard ratio.

placebo-treated controls ( 890 subjects with biomarker data at both enrolment and 3 months; $\mathrm{p}=0.02$ for the interaction term while controlling for clinic, $\mathrm{p}=0.018$ while controlling for clinic and other potential confounders). Plotting change in sTNFR75 by quartiles, we found that any decline in sTNFR75 levels between 0 and 3 months (the lower two quartiles) identified subjects in whom azithromycin was superior to placebo after 3 months (fig. 1a and b). We found no other interaction between any of the other biomarkers and response to azithromycin.

Tumour necrosis factor (TNF) can bind to two receptors, TNF receptor type 1 (CD120a, p55/60 and TNFR55) and TNF receptor type 2 (CD120b, p75/80 and TNFR75), which we refer to here as TNFR55 and TNFR75, respectively. TNFR55 is expressed in most tissues, whereas TNFR75 is only found in cells of the immune system and endothelial cells [3] and, in particular, marks a "non-classical" subpopulation of monocytes [4]. Functionally, the soluble TNF receptors may either be endogenous inhibitors of the effects of TNF- $\alpha$ [5] or stabilise the bioactivity of TNF- $\alpha$ by preventing dissociation of homotrimeric TNF molecules to inactive monomers [6]. Prior studies of sTNFR75 in COPD show that sTNFR75 levels can serve as a marker of TNF- $\alpha$ levels [7], sTNFR75 levels are elevated in stable COPD [7] and sTNFR75 levels are elevated during COPD exacerbations but decrease with resolution of the exacerbation [8]. Finally, sTNFR75 can be elevated in conditions that may complicate COPD such as CHF [9] or bronchiectasis [10]. In this study, TNF- $\alpha$ was below the recommended dynamic range of our assay $\left(3.9-250 \mathrm{pg} \cdot \mathrm{mL}^{-1}\right)$ in a 
substantial proportion of pilot samples. Therefore, we chose to study sTNFR75. Our data expand the utility of measuring sTNFR75 in COPD by confirming its value as a prognostic biomarker for future risk for exacerbation in a large prospective study and by establishing its value as a biomarker of reduction in AECOPD risk in a randomised trial. sTNFR75 levels could have been affected in some subjects in our trial due to comorbid CHF or bronchiectasis (although both would have to have been subclinical as history was used to exclude patients with either condition at entry), because of concomitant medications (e.g. use of statins or $\beta$-blockers that can decrease sTNR75 levels in the setting of cardiac disease) or if elevated sTNFR75 levels persisted in any subjects who may have had exacerbations $>4$ weeks prior to enrolment. However, treatment allocation was randomised in this clinical trial and unmeasured comorbidities or medication effects should be randomised across patients treated with placebo versus azithromycin.

In summary, in a prospectively designed biomarker analysis in patients enrolled in a large randomised trial, we found that higher sTNFR75 levels at enrolment were associated with increased risk for AECOPDs in exacerbation-prone subjects and that declines in sTNF75 with treatment were associated with the beneficial effects of azithromycin. If these findings can be validated in future studies, plasma sTNFR75 levels may be useful in targeting azithromycin use in COPD. azithromycin http://ow.ly/pNK5T

Prescott G. Woodruff ${ }^{1,2}$, Wissam Chatila ${ }^{3}$, John E. Connett ${ }^{4}$, Gerard J. Criner $^{3}$, Jeffrey L. Curtis ${ }^{5,6}$, Mark T. Dransfield ${ }^{7}$, MeiLan K. Han $^{6}$, Stephen C. Lazarus ${ }^{1}$, Nathaniel Marchetti ${ }^{3}$, Thomas J. Rogers ${ }^{3}$, Paul D. Scanlon ${ }^{8}$, Don D. Sin ${ }^{9}$, Helen Voelker ${ }^{4}$, Christine Wendt ${ }^{10}$, and Richard K. Albert ${ }^{11,12}$ for the COPD Clinical Research Network

${ }^{1}$ Cardiovascular Research Institute, University of California, San Francisco, CA, ${ }^{2}$ Dept of Medicine, Division of Pulmonary and Critical Care Medicine, University of California, San Francisco, CA, ${ }^{3}$ Division of Pulmonary and Critical Care Medicine, Temple University, Philadelphia, PA, ${ }^{4}$ Division of Biostatistics, University of Minnesota, School of Public Health, Minneapolis, MN, ${ }^{5}$ Division of Pulmonary and Critical Care Medicine, Ann Arbor VA Health System, Ann Arbor, MI, ${ }^{6}$ Division of Pulmonary and Critical Care Medicine, University of Michigan, Ann Arbor, MI, ${ }^{7}$ Division of Pulmonary, Allergy and Critical Care Medicine, University of Alabama, Birmingham, AL, ${ }^{8}$ Division of Pulmonary and Critical Care Medicine, Mayo Clinic, Rochester, MN, ${ }^{10}$ Division of Pulmonary Allergy, Critical Care and Sleep Medicine, University of Minnesota, VA Medical Center, Minneapolis, MN, ${ }^{11}$ Dept of Medicine, Denver Health Medical Center, Denver, CO, and ${ }^{12}$ Division of Pulmonary Sciences and Critical Care Medicine, University of Colorado Denver, Aurora, CO, USA. ${ }^{9}$ UBC James Hogg Research Centre, Institute for Heart + Lung Health, St Paul's Hospital, Vancouver, BC, Canada.

Correspondence: P.G. Woodruff, Dept of Medicine, Division of Pulmonary and Critical Care Medicine, University of California, Box 0130, Rm HSE 1305, 513 Parnassus Ave., San Francisco, CA 94143, USA. E-mail: prescott.woodruff @ucsf.edu

Received: March 192013 | Accepted: Aug 162013 | First published online: Oct 172013

Support statement: This work was supported by the National Institute of Health-National Heart, Lung, and Blood Institute (grants U10 HL074407, U10 HL074408, U10 HL074409, U10 HL074416, U10 HL074418, U10 HL074422, U10 HL074424, U10 HL074428, U10 HL074431, U10 HL074439, and U10 HL074441). Members of the COPD Clinical Research Network and their specific grant information can be found in the acknowledgements section.

Conflict of interest: Disclosures can be found alongside the online version of this article at www.erj.ersjournals.com

Acknowledgements:

We appreciate the expert assistance of L. Hester, J. Hasday and the Maryland Cytokine Core Laboratory (Baltimore, MD, USA) for performance of the ELISAs. At some sites, General Clinical Research Centers (GCRC) were utilised; their M01 grants from the National Center for Research Resources (Bethesda, MD, USA) are listed below. Members of this COPD Clinical Research Network and their grant support are as follows. Brigham and Women's Hospital, Boston, MA, USA (affiliated sites: Reliant Medical Group, Worcester, MA, USA; West Roxbury Veterans Hospital, West Roxbury, MA, USA): J.J. Reilly Jr (Principal Investigator), G.R. Washko (Co-Principal Investigator), R. Rosiello and M.L. Moy (Investigators), C. Mayo, S. McQuaid Peterson, K. Allain, A. McDonald, B. Kniskern, V. Danilack and K. Matthess (Coordinators) (grant HL074428 and GCRC grant RR02635). Denver Health Medical Center, Denver, CO, USA (affiliated sites: National Jewish Medical and Research Center, Denver, CO, USA; University of Colorado, Boulder, CO, USA): R.K. Albert (Principal Investigator), B. Make (Co-Principal Investigator), M. Schwarz and C. Welch (Investigators), M. Gilmartin and C. Verano (Coordinators) (grant HL074409 and GCRC grant RR00051). Los Angeles Biomedical Research Institute at Harbor-UCLA Medical Center, Torrance, CA, USA: R. Casaburi, (Principal Investigator), J. Porszasz, (Investigator), R.D. Love, K. Norulak, R. Kiledjian and G. Garcia (Coordinators) (grant HL074407 and GCRC grant RR00425). Minnesota Veterans Research Institute, Minneapolis, MN, USA (affiliated sites: HealthPartners Research Foundation, Bloomington, MN, USA; Mayo Clinic, Rochester, MN, USA): D.E. Niewoehner (Principal Investigator), C. McEvoy, K.R. Rice and P.D. Scanlon (Co-Principal Investigators), C.B. Bourassa, P. Neuenfeldt and J. Hart (Coordinators) (grant 1U10-HL074416). Temple University, Philadelphia, PA, USA: G.J. Criner (Principal Investigator), N. Marchetti, A. Satti, V Kim, J Mamary and F. Cordova (Investigators), C. Grabianowski, D. Fehrle, H. Criner and G. Jones (Coordinators) (grant HL074408). G.J. Criner (Principal Investigator), W. Chatila, N. Marchetti, V. Kim, G. D’Alonzo, S. Krachman, F. Cordova, K. Brennan, N. Patel and J. Mamary (Investigators), 
D. Fehrle, C. Grabianowski and G. Jones (Coordinators) (grant HL074408). University of Alabama at Birmingham, Birmingham, AL, USA: W.C. Bailey and J.A.D. Cooper (Co-Principal Investigators), M.T. Dransfield, L.B. Gerald and P. O’Reilly (Investigators), S. Tidwell (Coordinator) (grant HL074418). University of California, San Francisco, CA, USA: S.C. Lazarus (Principal Investigator), H.A. Boushey and P.G. Woodruff (Investigators), M. Birch, R. Sakurai, K. Schardein, M. Dyjak and C. Nguyen (Coordinators) (1U10-HL074431). University of Maryland, Baltimore, MD, USA: S.M. Scharf (Principal Investigator), M. Alattar, P. Amelung, M. Cowan, J. Hanson, J. Hasday, A. Iacono, C. Shanholtz, N. Todd and A. Verceles (Investigators), T. Fitzgerald, W. Bell-Farrell and P. Wood (Coordinators) (grant HL074441 and GCRC grant RR16500). University of Michigan, Ann Arbor, MI, USA: F.J. Martinez (Principal Investigator), J.L. Curtis, M.K. Han, K.R. Flaherty, T. Standiford, S.E. Gay and T.E. Sisson (Investigators), D. Thompson, L. McCloskey, M. Christensen and D. White (Coordinators) (grant HL074422). University of Pittsburgh, Pittsburgh, PA, USA: F. Sciurba (Principal Investigator), J. Bon (Investigator), L. Kniolek, M. Pitaro and R. Folger (Coordinators) (grant HL074439 and GCRC grant RR00056). University of Minnesota (Data Coordinating Center), Minneapolis, MN, USA: J.E. Connett (Principal Investigator), N.R. Anthonisen (Steering Committee Chair), C. Wendt (Co-Principal Investigator), M. Skeans, W. Patrek, H. Voelker and S. Harnden (Coordinators) (grant 1U10- HL074424). Data and Safety Monitoring Board: B.B. Bender, S.F. Kelsey, J.R. Landis, B. Phillips, G.M. Turino, R. Veatch, A. Waldo, C. Champlin and A. Wanner (all National Heart, Lung, and Blood Institute, Bethesda, MD, USA). Protocol Review Committee: H.W. Kelly (University of New Mexico-Health Sciences Center, Albuquerque, NM, USA), J. Maurer (The Cleveland Clinic Foundation, Cleveland, OH, USA), A.J. McSweeny (Northwestern University Medical School, Chicago, IL, USA), R.M. Senior (Washington University School of Medicine, St Louis, MO, USA), E.A. Thom (George Washington University, Rockville, MD, USA), P.D. Wagner (University of California, San Diego. San Diego, CA, USA), R.L. ZuWallack (Saint Francis Hospital and Medical Center, Hartford, CT, USA). National Heart, Lung, and Blood Institute, Bethesda, MD, USA: G. Weinmann (Deputy Director, Division of Lung Diseases), T. Croxton (Director, Airway Biology and Disease Program), A. Punturieri (Program Officer) and M.P. Stylianou (Biostatistician).

\title{
References
}

1 Albert RK, Connett J, Bailey WC, et al. Azithromycin for prevention of exacerbations of COPD. N Engl J Med. 2011; 365: 689-698.

2 Niewoehner DE, Rice K, Cote C, et al. Prevention of exacerbations of chronic obstructive pulmonary disease with tiotropium, a once-daily inhaled anticholinergic bronchodilator: a randomized trial. Ann Intern Med 2005; 143: 317-326.

3 Aggarwal BB. Tumour necrosis factors receptor associated signalling molecules and their role in activation of apoptosis, JNK and NF-кB. Ann Rheum Dis 2000; 59: Suppl. 1, i6-i16.

4 Hijdra D, Vorselaars AD, Grutters JC, et al. Differential expression of TNFR1 (CD120a) and TNFR2 (CD120b) on subpopulations of human monocytes. J Inflamm (Lond) 2012; 9: 38.

5 Aderka D, Engelmann H, Wysenbeek AJ, et al. The possible role of tumor necrosis factor (TNF) and its natural inhibitors, the soluble-TNF receptors, in autoimmune diseases. Isr J Med Sci 1992; 28: 126-130.

6 Aderka D, Engelmann H, Maor Y, et al. Stabilization of the bioactivity of tumor necrosis factor by its soluble receptors. J Exp Med 1992; 175: 323-329.

7 Takabatake $\mathrm{N}$, Nakamura $\mathrm{H}$, Abe S, et al. The relationship between chronic hypoxemia and activation of the tumor necrosis factor-alpha system in patients with chronic obstructive pulmonary disease. Am J Respir Crit Care Med 2000; 161: 1179-1184.

8 Groenewegen $\mathrm{KH}$, Dentener MA, Wouters EF. Longitudinal follow-up of systemic inflammation after acute exacerbations of COPD. Respir Med 2007; 101: 2409-2415.

9 Nozaki N, Yamaguchi S, Shirakabe M, et al. Soluble tumor necrosis factor receptors are elevated in relation to severity of congestive heart failure. Jpn Circ J 1997; 61: 657-664.

10 Osika E, Cavaillon JM, Chadelat K, et al. Distinct sputum cytokine profiles in cystic fibrosis and other chronic inflammatory airway disease. Eur Respir J 1999; 14: 339-346.

\section{Pulmonary embolism risk stratification: where are we heading?}

\author{
To the Editor:
}

We read with great interest the paper by SANCHEZ et al. [1], in which the authors suggested that biomarkers and echocardiography findings would provide additional prognostic information to traditional risk models, such as the pulmonary embolism severity index (PESI), in normotensive acute pulmonary embolism (PE) patients. Patients were assigned to low- (PESI I-II), intermediate- (PESI III-IV) and high-risk classes (PESI $\mathrm{V}$ ) and further stratified according to right ventricle dysfunction markers (right ventricle/left ventricle ratio, and troponin and brain natriuretic peptide (BNP) levels). The authors reported that low-risk PESI patients without any right ventricle dysfunction markers had a significantly lower risk for adverse events than low-risk patients with abnormal right ventricle/left ventricle ratio, troponin and/or BNP levels. However, the same results were not found for the remaining PESI classes (intermediate and high risk). 